\title{
Logistic Regression Analysis of the Factors Involved in the Failure of Osseointegration and Survival of Dental Implants with an Internal Connection and Machined Collar: A 6-Year Retrospective Cohort Study
}

\author{
Aritza Brizuela-Velasco $\left(\mathbb{D},{ }^{1}\right.$ Ángel Álvarez-Arenal ${ }^{(D)},{ }^{1}$ Esteban Pérez-Pevida $\left(\mathbb{D},{ }^{2,3}\right.$ \\ Iker Bellanco-De La Pinta, ${ }^{1}$ Héctor De Llanos-Lanchares $\mathbb{D}^{1}{ }^{1}$ Ignacio González-González, ${ }^{1}$ \\ and Carolina Larrazábal-Morón (iD) \\ ${ }^{1}$ Department of Surgery and Medical-Surgical Specialties, Faculty of Medicine, University of Oviedo, Oviedo, Spain \\ ${ }^{2}$ Department of Surgery, Faculty of Medicine, University of Salamanca, Salamanca, Spain \\ ${ }^{3}$ Faculty of Health Sciences, Miguel de Cervantes European University, Valladolid, Spain \\ ${ }^{4}$ School of Dentistry, Catholic University San Vicente Martir of Valencia, Valencia, Spain \\ Correspondence should be addressed to Aritza Brizuela-Velasco; aritzabrizuela@hotmail.com
}

Received 16 May 2021; Revised 1 September 2021; Accepted 21 September 2021; Published 7 October 2021

Academic Editor: Iole Vozza

Copyright (c) 2021 Aritza Brizuela-Velasco et al. This is an open access article distributed under the Creative Commons Attribution License, which permits unrestricted use, distribution, and reproduction in any medium, provided the original work is properly cited.

\begin{abstract}
Background. Although the long-term success rate of dental implants is currently close to 95\%, it is necessary to provide more evidence on the factors related to the failure of osseointegration and survival. Purpose. To establish the risk factors associated with the failure of osseointegration and survival of dental implants with an internal connection and machined collar and to establish a predictive statistical model. Materials and Methods. An analytical, retrospective, and observational clinical study of a sample of 297 implants with a follow-up of up to 76 months. Independent variables related to the implant, patient, and surgical and rehabilitative procedures were identified. The dependent variables were failure of osseointegration and failure of implant survival after prosthetic loading. A survival analysis was carried out by applying the Kaplan-Meier model (significance for $p<0.05$ ). The log-rank test and the Cox regression analysis were applied to the factors that presented differences. Finally, the regression logit function was used to determine whether it is possible to predict the risk of implant failure according to the analyzed variables with the data obtained in this study. Results. The percentages of osseointegration and survival were 97.6 and $97.2 \%$, respectively. For osseointegration, there were significant differences according to gender $(p=0.048)$, and the risk of nonosseointegration was $85 \%$ lower in women. Regarding survival, the Cox analysis converged on only two factors, which were smoking and treatment with anticoagulant drugs. The risk of loss was multiplied by 18.3 for patients smoking more than 10 cigarettes per day and by 28.2 for patients treated with anticoagulants. Conclusions. The indicated risk factors should be considered, but the analysis of the results is not sufficient to create a predictive model.
\end{abstract}

\section{Introduction}

Although the rehabilitation of edentulism using dental implants has a high predictability, there are many factors that influence its prognosis [1]. These factors are related to the patient, the surgical approach or applied load, the implant used in each case or the final prosthetic rehabilitation, and can limit or have a negative impact on osseointegration or on implant survival [2-4].

Implant failure is defined as the total failure of the implant to fulfill its purpose (functional, aesthetic, or phonetic) due to biological or mechanical causes [5]. It can occur during or after the process of osseointegration, once the definitive prosthetic loading has been carried out and 
the implant is functioning. Two types of dependent variables are frequently assessed during the second period: implant survival, which exclusively indicates the permanence of the implant in the mouth, or implant success, which is based on implant and peri-implant soft tissue parameters [6]. It can be considered that survival indicates the likelihood of the implant to continue performing its function, while success indicates the likelihood that it will do so without causing additional complications.

The scientific literature on this topic describes a series of risk factors associated with the prognosis of implant rehabilitations.

On the one hand, the intrinsic factors of the patient and the presence of certain systemic pathologies, habits, and/or pharmacological treatments, as well as the characteristics of the implant placement area, are apparently the most significant determining factors for dental implant success and survival rates [7-9]. In this regard, the presence of cardiovascular disease, diabetes, osteoporosis, antibone resorption treatments, and radiotherapy can be risk factors. Even so, the health and psychosocial benefits of implantsupported rehabilitation outweigh the risks inherent to the treatment of elderly patients, who are the main beneficiaries of this type of treatment.

The characteristics of the implant and of its prosthetic connection can also play a crucial role in the prognosis; its macroscopic and surface properties can be decisive depending on the case [10-12]. In this regard, clinical studies suggest that internal connection maybe advantageous for marginal bone preservation and, therefore, for implant survival [13].

On the other hand, the use of an appropriate surgical technique depending on the intrinsic characteristics of each case, the adoption of a correct loading protocol, and the effective selection of the final prosthetic rehabilitation, both in terms of design and material, in addition to the used retention, have an impact on the prevention of long-term dental implant failure [14-16]. However, it should be pointed out that the existing evidence in this field is very limited.

Although the long-term success rate of dental implants is currently close to 95\% [17], the presence of multiple combinations of risk factors associated with implant loss should be considered to minimize the risk of failure of implant-supported rehabilitations. Finally, it is also necessary to provide more evidence on the factors related to the failure of osseointegration and survival, independently.

Therefore, the objectives of the present analytical retrospective clinical study were to assess the osseointegration and survival rates of a sample of implants with an internal connection and machined collar, to analyze and compare the risk factors associated with implant failure, and to compare the results with those of previously published studies on the topic.

\section{Material and Methods}

In this observational clinical study, we retrospectively analyzed a sample of patients undergoing rehabilitation with dental implants. All implants and prostheses were placed by the same operator (Brizuela-Velasco, A.), and the data on the analyzed variables were obtained from the medical records of the patients, from the day of implant placement and throughout the follow-up period.

In accordance with point 13 of the general principles of the WMA Declaration of Helsinki (ethical principles for medical research involving human subjects), this observational study does not require to be assessed by an ethics committee. Therefore, our study can be considered a posttrial authorization, in which the assignment of a patient to a specific therapeutic strategy is not decided in advance by the protocol of a trial, rather it is established by the usual practice of dentistry, and the decision to prescribe the procedure is clearly dissociated from the decision to include the patient in the study.

All patients treated from January 2013 to June 2014, with a follow-up of up to 6 years, were included in the study.

Therefore, the inclusion criteria correspond to the indications and contraindications for implant rehabilitation in that sample of patients: Patients who presented partial or total edentulism and who were periodontally healthy with good oral hygiene and without medication-related (taking intravenous bisphosphonates) or systemic (uncontrolled diabetes, immunosuppressed) contraindications.

All patients were treated with Tissue Level Klockner Essential Cone implants (Klockner Implant System, Madrid, Spain) with an internal connection using a Morse taper and an internal octagon (Figure 1). These implants are made of a commercially pure Titanium Grade 3 with surface modification by blasting alumina particles and acid etching. In their crestal portion, the implants have a machine-polished collar for soft tissue apposition that is available in two heights: $0.7 \mathrm{~mm}$ or $1.5 \mathrm{~mm}$ (Figure 2). The selection of the height was based on the patient's gingival biotype and aesthetic criteria determined by the clinician (Brizuela-Velasco, A.).

All the implants that were assessed and obtained good osseointegration had a follow-up of 59 (minimum) to 76 (maximum) months after implant placement (Figure 3).

The following independent variables were assessed: implant related-length $(\leq 8 \mathrm{~mm} / 10 \mathrm{~mm} / 12 \mathrm{~mm})$, diameter $(3.5 \mathrm{~mm} / 4 \mathrm{~mm} / 4.5 \mathrm{~mm})$, and type of machined collar $(0.7 \mathrm{~mm} / 1.5 \mathrm{~mm})$; patient related-age, gender (male/female), smoking habits (nonsmoker/smoker of $<10$ cigarettes per day/smoker of $>10$ cigarettes per day), previous systemic pathologies (yes/no), anticoagulant drugs (yes/no), arterial hypertension (yes/no), diabetes (yes/no), psychotropic drugs (yes/no), and oral bisphosphonates (yes/no); and related to the surgical and rehabilitative treatment-follow-up time in months, arch (upper/lower), implant position (incisors/canines/premolars/molars), surgical procedure (submerged implants/nonsubmerged implants with transmucosal cap), specific type of surgery (conventional drilling/immediate implant/transcrestal osteotome sinus floor elevation/simultaneous bone regeneration/location of previous regeneration), type of prosthesis (single crown/fixed partial prosthesis/overdenture), and prosthetic retention (cemented/screwed). All these variables were recorded by a single evaluator (Brizuela-Velasco, A.). 


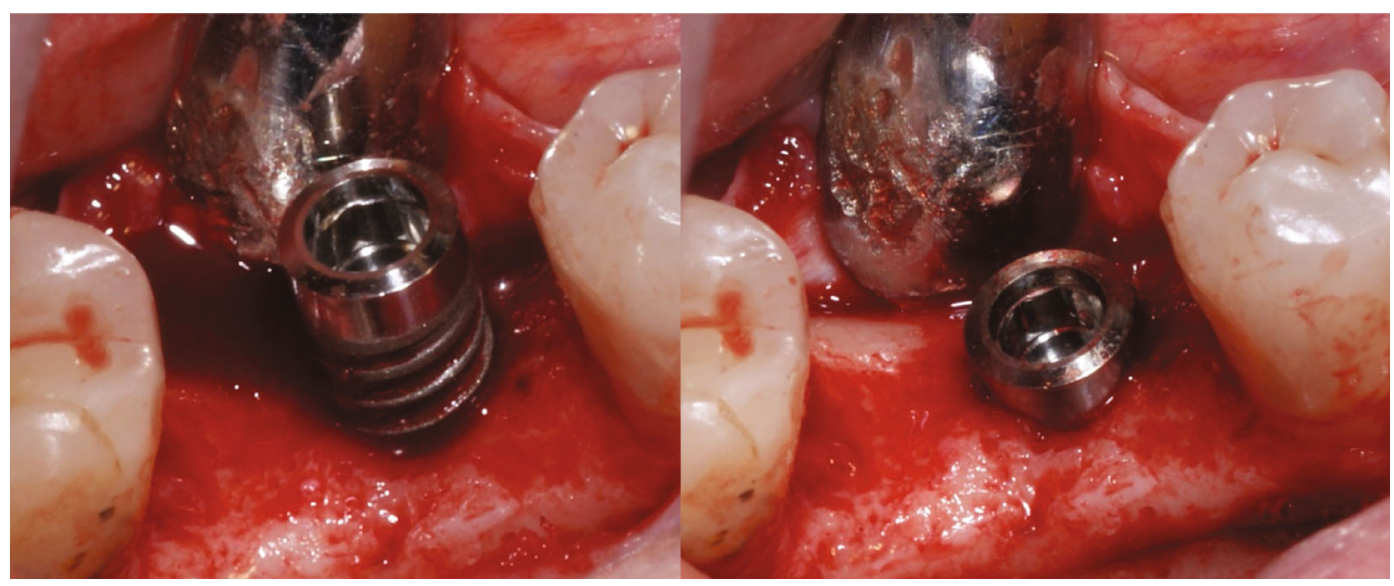

Figure 1: Surgical approach of the insertion of an Klockner Essential Cone implant with a machined collar of $1.5 \mathrm{~mm}$ height.

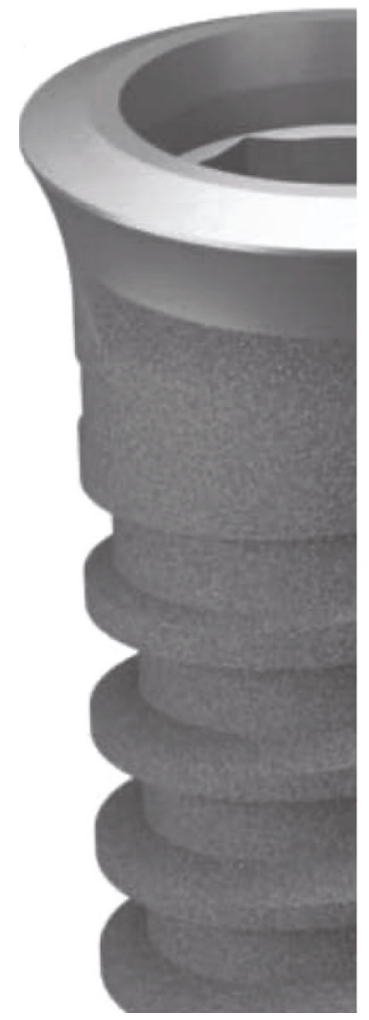

(a)

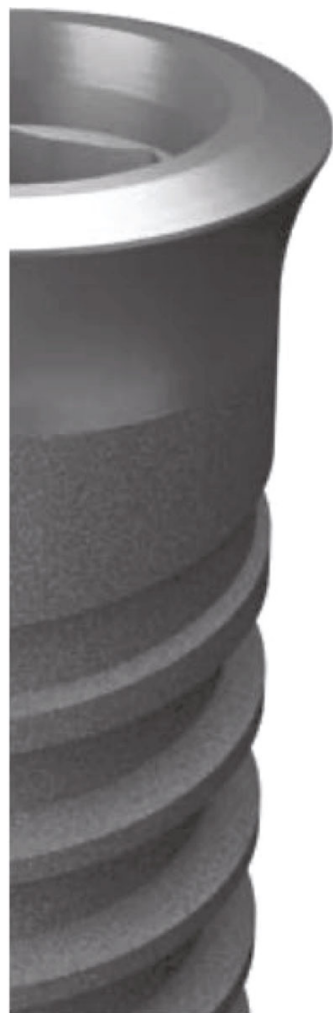

(b)

FIgURe 2: Design of the implants with a machined collar of $0.7 \mathrm{~mm}$ (a) and 1.5 (b) height.

The dependent variables analyzed were failure of osseointegration and implant failure after loading (no survival).

The SPSS 25.00 package (IBM SPSS Statistics, New York, USA) was used to carry out the statistical analysis. In addition to a descriptive analysis of the data obtained from each of the assessed variables, a survival analysis was performed by applying the Kaplan-Meier model to obtain the mean curve of survival months, with a $95 \%$ confidence interval and a statistical significance when $p<0.05$. The log-rank test was applied to analyze the significant differences in implant survival for the different studied factors. On the other hand, the Cox regression analysis was used to determine the implant survival risk rate for each factor of the study. The $p$ value of the Wald test was 0.05 at the start of the model and 0.1 at the end. Finally, the regression logit function was used to determine whether it is possible to predict the risk of implant failure according to the analyzed variables with the data obtained in this study. This study complies 


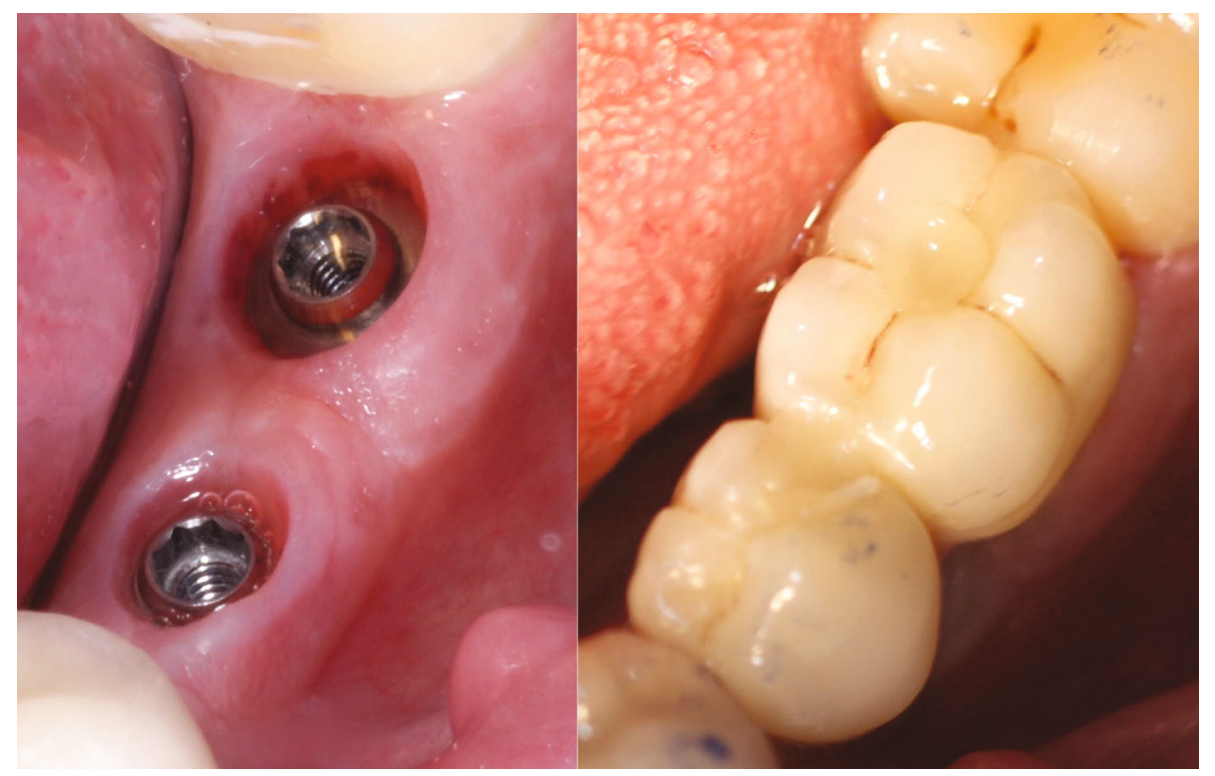

FIgURE 3: Screw-retained implant-supported prosthetic rehabilitation of two Klockner Essential Cone implants with a machined collar of $1.5 \mathrm{~mm}$ height.

with all checklist items of the statement of the STROBE Initiative (Strengthening the Reporting of Observational Studies in Epidemiology).

\section{Results}

This study assessed 297 implants in 110 patients with a mean age of $56.3( \pm 11.8)$. Tables 1 and 2 show the descriptive statistics of osseointegration failures and failures after loading, respectively, for each independent variable analyzed.

With a sample of 297 cases and to achieve a statistical power of $80 \%$, with a significance level of $5 \%$, will be considered as statistically significant an odds ratio of 0.5 when $p_{2} \leq p_{1}$ and 1.4 when $p_{2} \geq p_{1}$ provided that $P(Y=1 \mid X=$ $1)=0.2,0.7$ when $p_{2} \leq p_{1}$ and 1.5 when $p_{2} \geq p_{1}$ provided that $P(Y=1 \mid X=1)=0.5$, and 0.7 when $p_{2} \leq p_{1}$ and 2.7 when $p_{2} \geq p_{1}$ provided that $P(Y=1 \mid X=1)=0.7$.

The mean follow-up time was $64.5( \pm 11.7)$ months. There was osseointegration failure in 7 implants, which represents a success in $97.6 \%$ of the placed implants. In turn, 8 implants did not survive after loading, which represents a survival of $97.2 \%$ of the osseointegrated implants during the follow-up period.

3.1. Analysis of Nonosseointegrated Implants. The KaplanMeier model of survival and the log-rank test were applied to find significant differences for the assessed factors. The only factor for which there were significant differences was gender $(p=0.048)$, with a lower survival in men; that is, men had a lower percentage of osseointegration compared to women.

Regarding the Cox regression analysis, as predicted by the Kaplan-Meier model, there was no factor influencing osseointegration except gender, so no Cox model converged.
The logit estimation model showed that the risk of nonosseointegration was reduced by $85 \%$ in implants placed in women compared to men (Table 3).

3.2. Analysis of Nonsurviving Implants after Loading. The mean implant survival time was 73.4 months. Almost all implant losses occurred before 60 months; the survival was stable between 60 and 70 months of follow-up; finally, there was a slight decrease in survival at 75 months, with definitive survival percentages of approximately $97 \%$.

The log-rank test was applied to compare survival functions to determine whether there were significant differences in implant survival functions for the different factors. It should be noted that this comparison could not be performed for several factors for which there were no losses in any of the categories, such as failure after prosthetic loading, specific type of surgery, type of prosthesis, arterial hypertension, psychotropic drugs, and bisphosphonates.

There were significant differences in survival for the factors: gender, smoking, and anticoagulant drugs (Table 2).

The mean implant survival was 70.5 months in men and 75.3 months in women, and this was statistically different $(p \leq 0.001)$.

The mean survival time was $73.9,71.6$, and 68.9 months for nonsmokers, smokers of less than 10 cigarettes, and smokers of more than 10 cigarettes, respectively, with statistically significant differences $(p=0.049)$.

The mean implant survival period was 74.3 months in patients who were not on anticoagulant drugs and 66.4 for those who were, and this difference was statistically significant $(p=0.002)$.

The Cox regression analysis was used to analyze the survival risk rate for the different analyzed factors. The Cox analysis requires establishing a reference category for each factor, since it compares by how much the impact or 
TABLE 1: Descriptive analysis of the failure of osseointegration in order to the analyzed independent variables and survival analysis applying the Kaplan-Meier model.

\begin{tabular}{|c|c|c|c|c|c|c|c|c|}
\hline & & \multicolumn{7}{|c|}{ Osseointegration } \\
\hline & & \multicolumn{2}{|c|}{ Total } & \multicolumn{2}{|c|}{ Yes } & \multicolumn{2}{|c|}{ No } & \multirow{2}{*}{$\begin{array}{l}\text { Kaplan-Meier } \\
{ }^{*} \text { Sig. } p<0.05\end{array}$} \\
\hline & & Count & $\begin{array}{l}\text { Column } \\
(N, \%)\end{array}$ & Count & $\begin{array}{l}\text { Column } \\
(N, \%)\end{array}$ & Count & $\begin{array}{l}\text { Column } \\
(N, \%)\end{array}$ & \\
\hline \multirow{3}{*}{ Sex } & Total & 297 & $100.0 \%$ & 290 & $100.0 \%$ & 7 & $100.0 \%$ & \multirow{3}{*}{${ }^{*} 0.048$} \\
\hline & Male & 145 & $48.8 \%$ & 139 & $47.9 \%$ & 6 & $85.7 \%$ & \\
\hline & Female & 152 & $51.2 \%$ & 151 & $52.1 \%$ & 1 & $14.3 \%$ & \\
\hline \multirow{3}{*}{ Dental arch } & Total & 297 & $100.0 \%$ & 290 & $100.0 \%$ & 7 & $100.0 \%$ & \multirow{3}{*}{0.546} \\
\hline & Upper & 160 & $53.9 \%$ & 157 & $54.1 \%$ & 3 & $42.9 \%$ & \\
\hline & Lower & 137 & $46.1 \%$ & 133 & $45.9 \%$ & 4 & $57.1 \%$ & \\
\hline \multirow{5}{*}{$\begin{array}{l}\text { Implant position in the } \\
\text { dental arch }\end{array}$} & Total & 297 & $100.0 \%$ & 290 & $100.0 \%$ & 7 & $100.0 \%$ & \multirow{5}{*}{0.070} \\
\hline & Incisive & 39 & $13.1 \%$ & 38 & $13.1 \%$ & 1 & $14.3 \%$ & \\
\hline & Canine & 35 & $11.8 \%$ & 32 & $11.0 \%$ & 3 & $42.9 \%$ & \\
\hline & Premolar & 101 & $34.0 \%$ & 100 & $34.5 \%$ & 1 & $14.3 \%$ & \\
\hline & Molar & 122 & $41.1 \%$ & 120 & $41.4 \%$ & 2 & $28.6 \%$ & \\
\hline \multirow{5}{*}{ Implant length } & Total & 297 & $100.0 \%$ & 290 & $100.0 \%$ & 7 & $100.0 \%$ & \multirow{5}{*}{0.091} \\
\hline & 6.0 & 12 & $4.0 \%$ & 12 & $4.1 \%$ & 0 & $0.0 \%$ & \\
\hline & 8.0 & 109 & $36.7 \%$ & 104 & $35.9 \%$ & 5 & $71.4 \%$ & \\
\hline & 10.0 & 118 & $39.7 \%$ & 118 & $40.7 \%$ & 0 & $0.0 \%$ & \\
\hline & 12.0 & 58 & $19.5 \%$ & 56 & $19.3 \%$ & 2 & $28.6 \%$ & \\
\hline \multirow{4}{*}{ Implant diameter } & Total & 297 & $100.0 \%$ & 290 & $100.0 \%$ & 7 & $100.0 \%$ & \multirow{4}{*}{0.854} \\
\hline & 3.5 & 98 & $33.0 \%$ & 95 & $32.8 \%$ & 3 & $42.9 \%$ & \\
\hline & 4.0 & 145 & $48.8 \%$ & 142 & $49.0 \%$ & 3 & $42.9 \%$ & \\
\hline & 4.5 & 54 & $18.2 \%$ & 53 & $18.3 \%$ & 1 & $14.3 \%$ & \\
\hline \multirow{3}{*}{$\begin{array}{l}\text { Smooth polished neck } \\
\text { height }\end{array}$} & Total & 297 & $100.0 \%$ & 290 & $100.0 \%$ & 7 & $100.0 \%$ & \multirow{3}{*}{0.963} \\
\hline & $0.7 \mathrm{~mm}$ & 173 & $58.2 \%$ & 169 & $58.3 \%$ & 4 & $57.1 \%$ & \\
\hline & $1.5 \mathrm{~mm}$ & 124 & $41.8 \%$ & 121 & $41.7 \%$ & 3 & $42.9 \%$ & \\
\hline \multirow{6}{*}{ Surgical technique } & Total & 297 & $100.0 \%$ & 290 & $100.0 \%$ & 7 & $100.0 \%$ & \multirow{6}{*}{0.633} \\
\hline & Conventional drilling mature bone & 219 & $73.7 \%$ & 214 & $73.8 \%$ & 5 & $71.4 \%$ & \\
\hline & Postdental exodontia & 36 & $12.1 \%$ & 34 & $11.7 \%$ & 2 & $28.6 \%$ & \\
\hline & Atraumatic sinus lift & 17 & $5.7 \%$ & 17 & $5.9 \%$ & 0 & $0.0 \%$ & \\
\hline & Simultaneous bone regeneration & 20 & $6.7 \%$ & 20 & $6.9 \%$ & 0 & $0.0 \%$ & \\
\hline & $\begin{array}{l}\text { Located in previously regenerated } \\
\text { bone }\end{array}$ & 5 & $1.7 \%$ & 5 & $1.7 \%$ & 0 & $0.0 \%$ & \\
\hline \multirow{3}{*}{ Surgical phases } & Total & 297 & $100.0 \%$ & 290 & $100.0 \%$ & 7 & $100.0 \%$ & \multirow{3}{*}{0.479} \\
\hline & One phase & 173 & $58.2 \%$ & 168 & $57.9 \%$ & 5 & $71.4 \%$ & \\
\hline & Two phases & 124 & $41.8 \%$ & 122 & $42.1 \%$ & 2 & $28.6 \%$ & \\
\hline \multirow{4}{*}{ Smoker } & Total & 297 & $100.0 \%$ & 290 & $100.0 \%$ & 7 & $100.0 \%$ & \\
\hline & No & 231 & $77.8 \%$ & 227 & $78.3 \%$ & 4 & $57.1 \%$ & 0242 \\
\hline & $<10 \mathrm{cig} /$ day & 36 & $12.1 \%$ & 35 & $12.1 \%$ & 1 & $14.3 \%$ & 0.242 \\
\hline & $>10 \mathrm{cig} /$ day & 30 & $10.1 \%$ & 28 & $9.7 \%$ & 2 & $28.6 \%$ & \\
\hline & Total & 297 & $100.0 \%$ & 290 & $100.0 \%$ & 7 & $100.0 \%$ & \\
\hline Previous medical conditions & No & 204 & $68.7 \%$ & 200 & $69.0 \%$ & 4 & $57.1 \%$ & 0.498 \\
\hline & Yes & 93 & $31.3 \%$ & 90 & $31.0 \%$ & 3 & $42.9 \%$ & \\
\hline & Total & 297 & $100.0 \%$ & 290 & $100.0 \%$ & 7 & $100.0 \%$ & \\
\hline Anticoagulant drugs & No & 251 & $84.5 \%$ & 246 & $84.8 \%$ & 5 & $71.4 \%$ & 0.327 \\
\hline & Yes & 46 & $15.5 \%$ & 44 & $15.2 \%$ & 2 & $28.6 \%$ & \\
\hline
\end{tabular}


TABLE 1: Continued.

\begin{tabular}{|c|c|c|c|c|c|c|c|c|}
\hline & & & & & Osseointe & gration & & \\
\hline & & & otal & & Yes & & No & Kaplan-Meier \\
\hline & & Count & $\begin{array}{c}\text { Column } \\
(N, \%)\end{array}$ & Count & $\begin{array}{c}\text { Column } \\
(N, \%)\end{array}$ & Count & $\begin{array}{c}\text { Column } \\
(N, \%)\end{array}$ & ${ }^{*}$ Sig. $p<0.05$ \\
\hline & Total & 297 & $100.0 \%$ & 290 & $100.0 \%$ & 7 & $100.0 \%$ & \\
\hline Arterial hypertension & No & 272 & $91.6 \%$ & 265 & $91.4 \%$ & 7 & $100.0 \%$ & 0.419 \\
\hline & Yes & 25 & $8.4 \%$ & 25 & $8.6 \%$ & 0 & $0.0 \%$ & \\
\hline & Total & 297 & $100.0 \%$ & 290 & $100.0 \%$ & 7 & $100.0 \%$ & \\
\hline Diabetes & No & 286 & $96.3 \%$ & 280 & $96.6 \%$ & 6 & $85.7 \%$ & 0.126 \\
\hline & Yes & 11 & $3.7 \%$ & 10 & $3.4 \%$ & 1 & $14.3 \%$ & \\
\hline & Total & 297 & $100.0 \%$ & 290 & $100.0 \%$ & 7 & $100.0 \%$ & \\
\hline Psychoactive drugs & No & 272 & $91.6 \%$ & 265 & $91.4 \%$ & 7 & $100.0 \%$ & 0.419 \\
\hline & Yes & 25 & $8.4 \%$ & 25 & $8.6 \%$ & 0 & $0.0 \%$ & \\
\hline & Total & 297 & $100.0 \%$ & 290 & $100.0 \%$ & 7 & $100.0 \%$ & \\
\hline Bisphosphonate drugs & No & 291 & $98.0 \%$ & 284 & $97.9 \%$ & 7 & $100.0 \%$ & 0.702 \\
\hline & Yes & 6 & $2.0 \%$ & 6 & $2.1 \%$ & 0 & $0.0 \%$ & \\
\hline
\end{tabular}

risk rate is multiplied if the implant belongs to a given category with respect to the reference. In the case of this study, the first category of each factor was considered as the reference.

After performing the Cox analysis, the model converged on only two factors, smoking and treatment with anticoagulant drugs. This means that these variables produce significant differences in the survival risk rate or loss rate (Table 4).

In this regard, the survival time decreased by $4.2 \%$ in patients who smoked more than 10 cigarettes per day when compared with those who did not smoke.

On the other hand, the survival time decreased by $4.4 \%$ in patients on anticoagulants when compared with those not on these drugs.

Finally, the logit estimation model could detect those predictors that influence the risk of implant loss and their impact has been quantified.

It was thus determined that the factors that influenced survival also predicted the risk of implant loss; it was multiplied by 18.3 for patients who smoked more than 10 cigarettes per day and by 28.2 for patients on anticoagulants.

The logistic regression models also provided a classification or prognosis of the implants (whether they will be lost or not) based on the estimated probability. To do this, a probability 0.1 was selected as the cut-off point that provides the optimum correction percentages for the study. Any implant with a probability of $>0.1$ was classified as lost.

The results demonstrated that our study adequately classified the implants, both intact and lost (sensitivity and specificity). However, it was not a good predictive model for loss since $88.5 \%$ of the implants diagnosed as lost were found to be intact. Therefore, it is necessary to look for more potential factors of implant loss to establish a predictive model.

\section{Discussion}

The objective of this retrospective clinical study was to assess the success of osseointegration, the survival of implants with an internal connection and machined collar, and to analyze the impact of risk factors associated with implant failure.

The study included a sample of 297 implants, and osseointegration failure occurred in 7 cases, which represents an osseointegration success rate of $97.6 \%$. These results are similar to those of previous studies, which reported that implants have an osseointegration success rate of 71.4 to 98.7\% depending on their location within the arches [18].

On the other hand, 8 implants of the final sample of 290 implants assessed after loading failed, which represents a survival of $97.2 \%$ during the follow-up period of up to 76 months. These results agree with the findings of a systematic review of the literature that included 23 studies and 7711 implants and revealed that implant survival rate in a 10year follow-up is approximately 95\% [19].

To meet the objectives of this study, an inferential statistic was performed to compare and establish the probability of osseointegration failure and survival for the dependent variables related to the implant, patient, and procedure.

4.1. Variables Related to the Implant. The log rank test found no differences in osseointegration and survival for the following implant variables: length, diameter, and height of the machined collar. However, for osseointegration, due to the proximity of the $p$ value to the acceptance threshold $(p=0.091)$, there was a trend for osseointegration to be greater in lengths of $10 \mathrm{~mm}$ compared to the other lengths.

It is believed that the length of the implant can affect osseointegration since it can be correlated with the possibility of obtaining a greater primary stability. On the other hand, the implant diameter and the height of the machined collar should be more relevant during loading, one for determining the possibilities of stress dissipation and the other for its relationship with the maintenance of a correct biological space. However, the meta-analyses that assessed the relationship of the length and diameter of the implant with its survival did not find statistically significant differences 
TABLE 2: Descriptive analysis of the failure of survival (after loading) in order to the analyzed independent variables and survival analysis applying the Kaplan-Meier model.

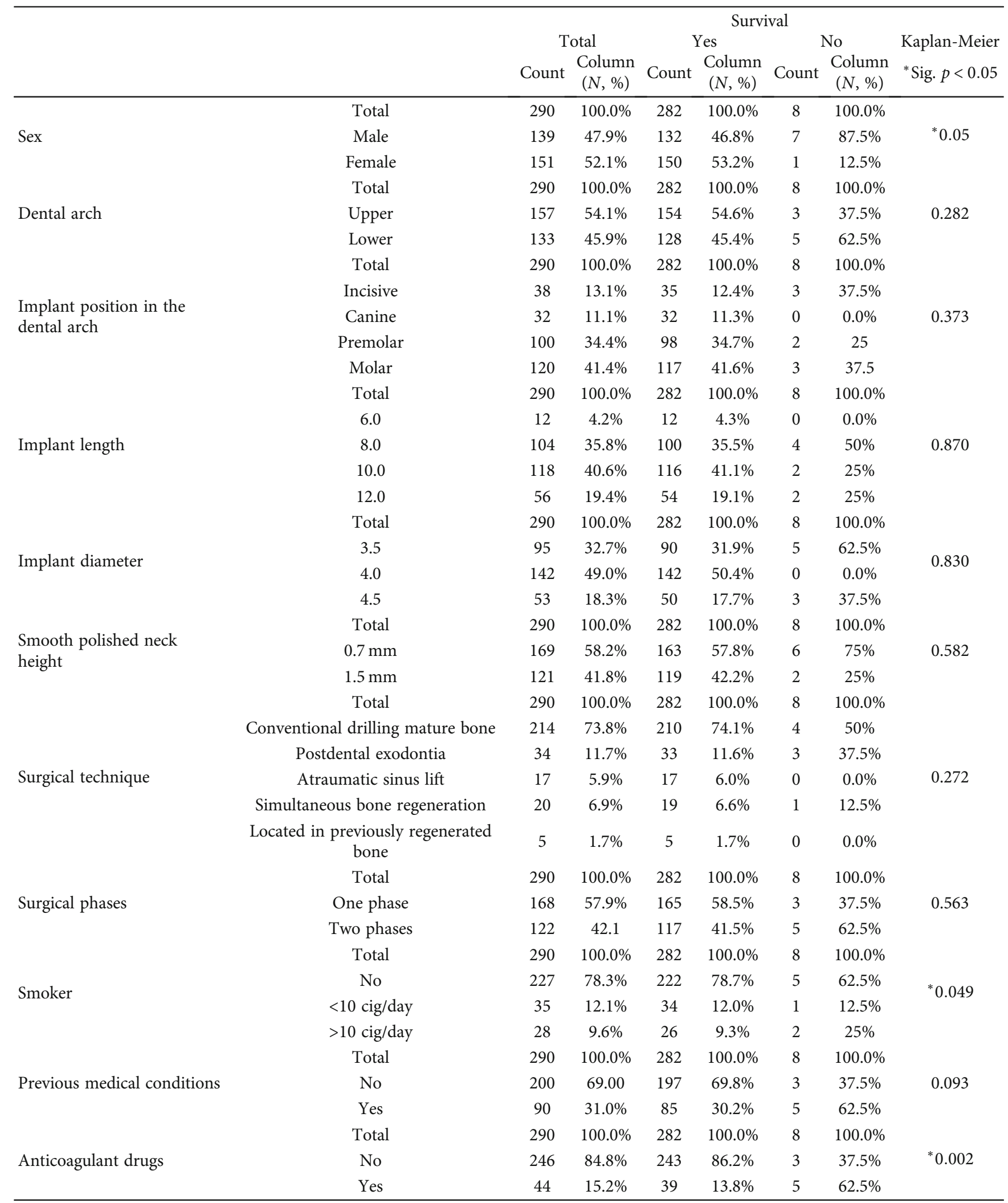


TABLE 2: Continued.

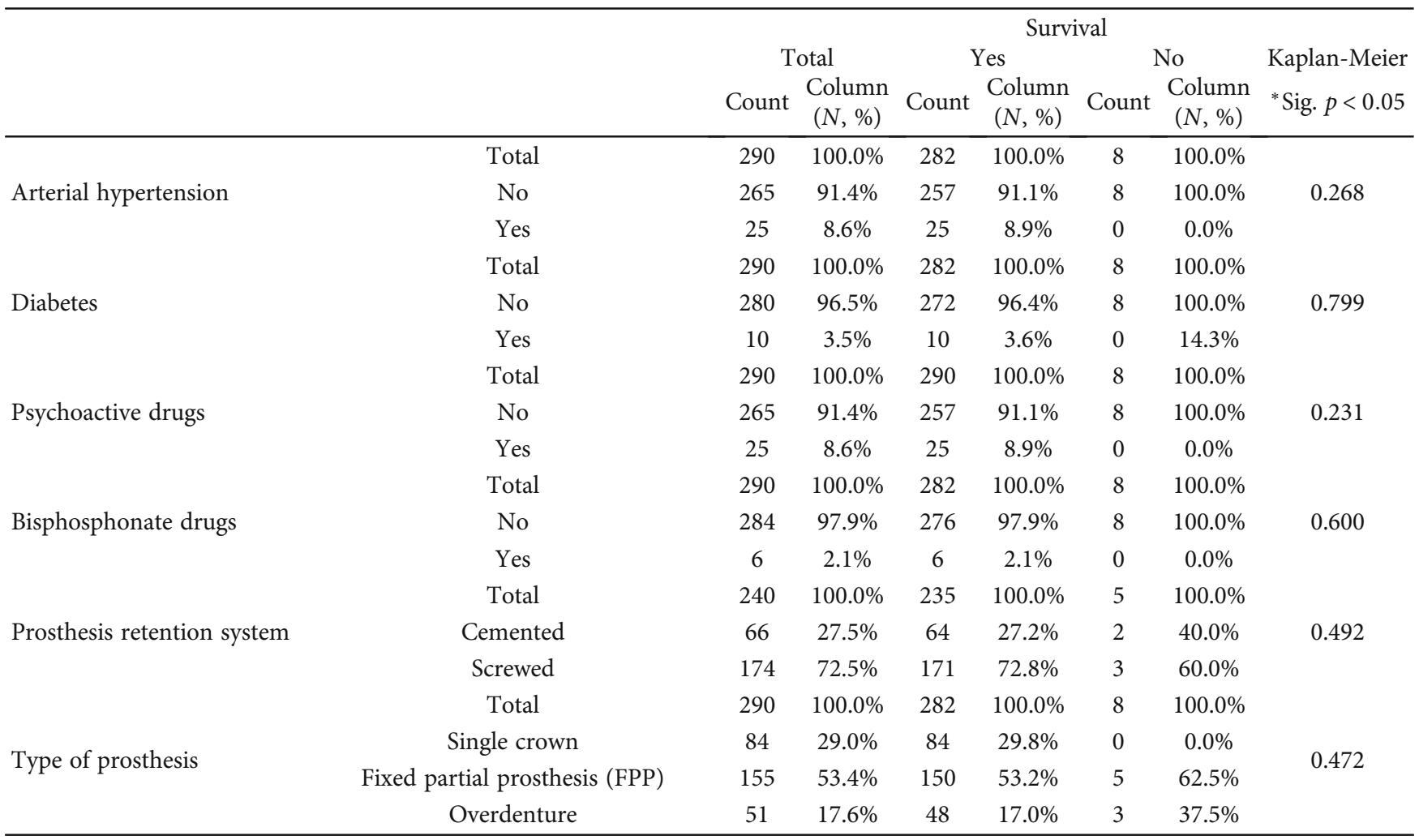

*Statistically significant findings.

TABLE 3: Results of the logit estimation model for nonosseointegration for risk factor sex, with a $10 \%$ significance level ( $p$ value $<0.1$ ). Odds ratio $=p /(1-p)=0.045 * 0.149^{\text {Sex }}$.

\begin{tabular}{ccccccccc}
\hline & \multirow{2}{*}{ S.E. } & \multirow{2}{*}{ Wald } & df & Sig. & \multicolumn{2}{c}{ Exp (B) } & $\begin{array}{r}\text { 90\% CI for Exp (B) } \\
\text { Lower }\end{array}$ \\
\hline \multirow{2}{*}{ Step $1^{\mathrm{a}}$} & Sex: female & -1.905 & 1.087 & 3.072 & 1 & 0.080 & 0.149 & 0.025 \\
& Constant & -3.106 & 0.417 & 55.406 & 1 & 0.000 & 0.045 & 0.889 \\
\hline
\end{tabular}

TABLE 4: Cox regression analysis for the dependent variable failure of survival.

\begin{tabular}{|c|c|c|c|c|c|c|c|c|c|}
\hline & & \multirow[t]{2}{*}{$B$} & \multirow[t]{2}{*}{ SE } & \multirow[t]{2}{*}{ Wald } & \multirow[t]{2}{*}{$d f$} & \multirow[t]{2}{*}{ Sig. } & \multirow[t]{2}{*}{$\operatorname{Exp}(B)$} & \multicolumn{2}{|c|}{$\begin{array}{l}95.0 \% \mathrm{CI} \text { for } \\
\operatorname{Exp}(B)\end{array}$} \\
\hline & & & & & & & & Lower & Upper \\
\hline \multirow[t]{2}{*}{ Pass 1} & Anticoagulant: yes (cat. ref. no) & 2.050 & 0.742 & 7.621 & 1 & 0.006 & 7.764 & 1.812 & 33.268 \\
\hline & Smoker: no (cat. ref.) & & & 5.747 & 2 & 0.056 & & & \\
\hline \multirow{3}{*}{ Pass 2} & Smoker: $<10 \mathrm{cig} /$ day & 1.022 & 1.138 & 0.807 & 1 & 0.369 & 2.780 & 0.299 & 25.843 \\
\hline & Smoker: >10 cig/day & 3.117 & 1.302 & 5.729 & 1 & ${ }^{*} 0.017$ & 22.585 & 1.759 & 289.996 \\
\hline & Anticoagulant & 3.170 & 1.124 & 7.957 & 1 & ${ }^{*} 0.005$ & 23.814 & 2.632 & 215.508 \\
\hline
\end{tabular}

$H(t)=H 0(t) * 22.585^{>10 \text { cig/day }} * 23.814^{\text {Anticoagulant }}{ }^{*}$ Statistically significant findings.

$[20,21]$. Similarly, there are no survival studies in the literature that compare different heights of the machined collar, although there are controlled and randomized clinical trials that show similar results for both marginal bone loss and long-term prognosis [22].
4.2. Variables Related to the Patient. There were only three factors in the present study that showed statistically significant differences regarding their influence on implant survival. Furthermore, two of them, smoking and anticoagulants, were indicative of a higher risk of implant failure. 
As for smoking, there was a decrease of $4.2 \%$ in the survival rate of patients who smoked more than 10 cigarettes per day and the risk of implant failure was multiplied by 18.3. Similar results are found in most of the survival studies analyzed in systematic reviews and meta-analyses, which conclude that smoking significantly reduces long-term success and survival rates [23-25]. This is mainly due to the vasoconstrictor properties of tobacco, which hinder proper vascularization and clot formation in the initial phases of osseointegration. Furthermore, smoking is usually associated with a lower level of oral hygiene, thus increasing the risk of dental implant failure [25].

The implants placed in patients on anticoagulants showed a $4.4 \%$ decrease in the survival rate, and the risk of implant loss was multiplied by 28.2 . The implant survival studies that analyzed the administration of oral anticoagulants as a risk factor for failure did not find significant differences between patients with and without cardiovascular disease. However, there is a higher percentage of implant loss in anticoagulated patients. According to these studies, the administration of anticoagulants does not seem to influence survival by itself, but it is a potential risk factor, particularly in elderly patients with chronic systemic pathologies and long-term pharmacological treatments [7, 26]. This finding agrees with the results obtained in our study, since all patients treated with oral anticoagulants suffered from some other systemic pathology and were older than the mean age. Therefore, age and comorbidities seem to be stronger determining factors for implant success and survival than the administration of specific drugs; this may be attributed to the lack of autonomy and insufficient oral care in older and comorbid patients [7].

The last factor influencing implant survival time was gender. With a survival of 70.5 months in men and 75.3 in women, the difference was significant. Nevertheless, it was not established as a risk factor for implant failure. These results are consistent with those of several retrospective clinical studies that show a higher short- and long-term implant failure rate for men compared to women $[27,28]$ and can be attributed to a higher consumption of tobacco and less concern for oral hygiene in men compared to women, although the evidence in this regard is limited.

Finally, there was no osseointegration in 7 implants of the present study before prosthetic rehabilitation, and these were independently analyzed to assess potential risk factors for this event. The only factor showing significant differences in survival was gender; osseointegration was statistically lower in men than in women. Similar retrospective studies agree with these results, showing a higher rate of early implant failure in men [29].

However, the regression logit function revealed that it is not possible to develop a predictive model with the data obtained in this retrospective clinical study.

\subsection{Variables Related to the Surgical and Rehabilitative} Procedure. Again, the log rank test found no differences in osseointegration and survival related to the different variables of the surgical or rehabilitative procedure. However, some clinical studies observe important differences in osseointegration related to the position in the arch, namely, the study of Drago [18] that shows values ranging from $71.4 \%$ in the posterior maxilla to $98.7 \%$ in the anterior mandible. The surgical placement protocol (one-stage vs. two-stage) deserves a special mention. A 2009 metaanalysis concluded that no statistically significant differences were observed between the two procedures; however, trends suggested less implant failures with the 2-stage (submerged) approach especially in fully edentulous patients [30]. Finally, another controversial factor related to implant survival is the type of retention: screwed or cemented. The 2015 metaanalysis of Lemos et al. found higher survival rates and lower marginal bone loss for cemented prostheses and higher prosthetic complications for screwed prostheses. However, the authors considered that the differences were not clinically significant [31].

\section{Conclusions}

Considering the intrinsic limitations of this study, the following conclusions can be drawn:

(i) The internal connection and machined collar implants have a high rate of osseointegration (97.6\%) and survival (97.2\%) in a follow-up period of up to 76 months

(ii) The male gender is associated with more osseointegration failures

(iii) The male gender, smoking more than 10 cigarettes per day, and anticoagulant treatment seem to influence survival after loading of dental implants

(iv) These conclusions should be considered with caution since the results are not sufficient to develop a predictive model and it is necessary to look for more potential factors responsible for failure

\section{Data Availability}

The data could be provided under request.

\section{Additional Points}

Summary Box. What is known: Long-term success rate of dental implants is close to $95 \%$; risk factors associated with implant loss should be considered. It is also necessary to provide more evidence on the factors related to the failure of osseointegration and survival. What this study adds: This study ratifies the most prevalent risk factors related to implant failure and underlines the significance of those factors related to the patient.

\section{Conflicts of Interest}

The authors declare no conflict of interest. 


\section{Authors' Contributions}

A.B-V. is responsible for the concept/design and data analysis/interpretation. Á.Á-A. is responsible for the critical revision of the article. E.P-P. is responsible for the writing/drafting the article. I.B-D. is responsible for the data collection. H.D-L. is responsible for the data collection and statistics. I.G-G. is responsible for the data collection. C.L-M. is responsible for the data analysis/interpretation and approval of the article.

\section{References}

[1] M. Srinivasan, S. Meyer, A. Mombelli, and F. Müller, "Dental implants in the elderly population: a systematic review and meta-analysis," Clinical Oral Implants Research, vol. 28, no. 8, pp. 920-930, 2017.

[2] O. Geckili, H. Bilhan, E. Geckili, A. Cilingir, E. Mumcu, and C. Bural, "Evaluation of possible prognostic factors for the success, survival, and failure of dental implants," Implant Dentistry, vol. 23, no. 1, pp. 44-50, 2014.

[3] K. Noda, H. Arakawa, A. Kimura-Ono et al., "A longitudinal retrospective study of the analysis of the risk factors of implant failure by the application of generalized estimating equations," Journal of Prosthodontic Research, vol. 59, no. 3, pp. 178-184, 2015.

[4] L. J. Heitz-Mayfield, M. Aaboe, M. Araujo et al., "Group 4 ITI consensus report: risks and biologic complications associated with implant dentistry," Clinical Oral Implants Research, vol. 29, no. S16, pp. 351-358, 2018.

[5] A. S. el Askary, R. M. Meffert, and T. Griffin, "Why do dental implants fail? Part I," Implant Dentistry, vol. 8, no. 2, pp. 173185, 1999.

[6] P. Papaspyridakos, C. J. Chen, M. Singh, H. P. Weber, and G. O. Gallucci, "Success criteria in implant dentistry: a systematic review," Journal of Dental Research, vol. 91, no. 3, pp. 242248, 2012.

[7] M. Schimmel, M. Srinivasan, G. McKenna, and F. Müller, "Effect of advanced age and/or systemic medical conditions on dental implant survival: a systematic review and meta-analysis," Clinical Oral Implants Research, vol. 29, Supplement 16, pp. 311-330, 2018.

[8] B. R. Chrcanovic, T. Albrektsson, and A. Wennerberg, "Bone quality and quantity and dental implant failure: a systematic review and meta-analysis," The International Journal of Prosthodontics, vol. 30, no. 3, pp. 219-237, 2017.

[9] W. Martin, E. Lewis, and A. Nicol, "Local risk factors for implant therapy," The International Journal of Oral \& Maxillofacial Implants, vol. 24, pp. 28-38, 2009.

[10] R. E. Jung, B. al-Nawas, M. Araujo et al., "Group 1 ITI consensus report: the influence of implant length and design and medications on clinical and patient-reported outcomes," Clinical Oral Implants Research, vol. 29, Supplement 16, pp. 69-77, 2018.

[11] H. de Bruyn, V. Christiaens, R. Doornewaard et al., "Implant surface roughness and patient factors on long-term periimplant bone loss," Periodontol 2000, vol. 73, no. 1, pp. 218227, 2017.

[12] C. A. A. Lemos, F. R. Verri, E. A. Bonfante, J. F. Santiago Júnior, and E. P. Pellizzer, "Comparison of external and internal implant-abutment connections for implant supported prostheses. A systematic review and meta-analysis," Journal of Dentistry, vol. 70, pp. 14-22, 2018.

[13] R. Caricasulo, L. Malchiodi, P. Ghensi, G. Fantozzi, and A. Cucchi, "The influence of implant-abutment connection to peri-implant bone loss: a systematic review and meta-analysis," Clinical Implant Dentistry and Related Research, vol. 20, no. 4, pp. 653-664, 2018.

[14] G. Troiano, L. Lo Russo, L. Canullo, D. Ciavarella, L. Lo Muzio, and L. Laino, "Early and late implant failure of submerged versus non-submerged implant healing: a systematic review, meta-analysis and trial sequential analysis," Journal of Clinical Periodontology, vol. 45, no. 5, pp. 613-623, 2018.

[15] M. Esposito, M. G. Grusovin, I. P. Polyzos, P. Felice, and H. V. Worthington, "Timing of implant placement after tooth extraction: immediate, immediate-delayed or delayed implants? A Cochrane systematic review," European Journal of Oral Implantology, vol. 3, no. 3, pp. 189-205, 2010.

[16] P. Pesce, L. Canullo, M. G. Grusovin, H. de Bruyn, J. Cosyn, and P. Pera, "Systematic review of some prosthetic risk factors for periimplantitis," The Journal of Prosthetic Dentistry, vol. 114, no. 3, pp. 346-350, 2015.

[17] M. S. Howe, W. Keys, and D. Richards, "Long-term (10-year) dental implant survival: a systematic review and sensitivity meta-analysis," Journal of Dentistry, vol. 84, pp. 9-21, 2019.

[18] C. J. Drago, "Rates of osseointegration of dental implants with regard to anatomical location," Journal of Prosthodontics, vol. 1, no. 1, pp. 29-31, 1992.

[19] V. Moraschini, L. A. C. Poubel, V. F. Ferreira, and E. S. P. Barboza, "Evaluation of survival and success rates of dental implants reported in longitudinal studies with a follow-up period of at least 10 years: a systematic review," International Journal of Oral and Maxillofacial Surgery, vol. 44, no. 3, pp. 377-388, 2015.

[20] H. N. Parize, L. O. L. Bohner, L. T. Gama et al., "Narrow-diameter implants in the anterior region: a meta-analysis," The International Journal of Oral \& Maxillofacial Implants, vol. 34, no. 6, pp. 1347-1358, 2019.

[21] P. Papaspyridakos, A. De Souza, K. Vazouras, H. Gholami, S. Pagni, and H. P. Weber, "Survival rates of short dental implants $(\leq 6 \mathrm{~mm})$ compared with implants longer than 6 $\mathrm{mm}$ in posterior jaw areas: a meta-analysis," Clinical Oral Implants Research, vol. 16, pp. 8-20, 2018.

[22] M. Herrero-Climent, M. Ruiz, C. Díaz-Castro, P. Bullón, and J. Ríos-Santos, "Influence of two different machined-collar heights on crestal bone loss," The International Journal of Oral \& Maxillofacial Implants, vol. 29, no. 6, pp. 13741379, 2014.

[23] V. Moraschini and E. S. P. Barboza, "Success of dental implants in smokers and non-smokers: a systematic review and meta-analysis," International Journal of Oral and Maxillofacial Surgery, vol. 45, no. 2, pp. 205-215, 2016.

[24] A. Veitz-Keenan, "Marginal bone loss and dental implant failure may be increased in smokers," Evidence-Based Dentistry, vol. 17, no. 1, pp. 6-7, 2016.

[25] S. A. Alfadda, "Current evidence on dental implants outcomes in smokers and nonsmokers: a systematic review and metaanalysis," The Journal of Oral Implantology, vol. 44, pp. 390399, 2018.

[26] A. Mombelli and N. Cionca, "Systemic diseases affecting osseointegration therapy," Clinical Oral Implants Research, vol. 17, no. S2, pp. 97-103, 2006. 
[27] M. V. Olmedo-Gaya, F. J. Manzano-Moreno, E. CañaveralCavero, J. de Dios Luna-del Castillo, and M. Vallecillo-Capilla, "Risk factors associated with early implant failure: a 5-year retrospective clinical study," The Journal of Prosthetic Dentistry, vol. 115, no. 2, pp. 150-155, 2016.

[28] K. Grisar, D. Sinha, J. Schoenaers, T. Dormaar, and C. Politis, "Retrospective analysis of dental implants placed between 2012 and 2014: indications, risk factors, and early survival," The International Journal of Oral \& Maxillofacial Implants, vol. 32, no. 3, pp. 649-654, 2017.

[29] G. Lin, S. Ye, F. Liu, and F. He, "A retrospective study of 30,959 implants: risk factors associated with early and late implant loss," Journal of Clinical Periodontology, vol. 45, no. 6, pp. 733-743, 2018.

[30] M. Esposito, M. G. Grusovin, Y. S. Chew, P. Coulthard, and H. V. Worthington, "Interventions for replacing missing teeth: 1- versus 2-stage implant placement," Cochrane Database of Systematic Reviews, vol. 3, article CD006698, 2009.

[31] C. A. Lemos, V. E. de Souza Batista, D. A. F. Almeida, J. F. Santiago Júnior, F. R. Verri, and E. P. Pellizzer, "Evaluation of cement-retained versus screw-retained implant-supported restorations for marginal bone loss: a systematic review and meta-analysis," The Journal of Prosthetic Dentistry, vol. 115, no. 4, pp. 419-427, 2016. 